\title{
Consistent conformal extensions of the standard model
}

\author{
Florian Loebbert, ${ }^{1, *}$ Julian Miczajka, ${ }^{1, \dagger}$ and Jan Plefka ${ }^{1,2, \$}$ \\ ${ }^{1}$ Institut für Physik, Humboldt-Universität zu Berlin, Zum Großen Windkanal 6, 12489 Berlin, Germany \\ ${ }^{2}$ Theoretical Physics Department, CERN, 1211 Geneva 23, Switzerland
}

(Received 15 September 2018; published 22 January 2019)

\begin{abstract}
The question of whether classically conformal modifications of the standard model are consistent with experimental observations has recently been subject to renewed interest. The method of Gildener and Weinberg provides a natural framework for the study of the effective potential of the resulting multiscalar standard model extensions. This approach relies on the assumption of the ordinary loop hierarchy $\lambda_{\mathrm{s}} \sim g_{\mathrm{g}}^{2}$ of scalar and gauge couplings. On the other hand, Andreassen et al. recently argued that in the (singlescalar) standard model gauge invariant results require the consistent scaling $\lambda_{\mathrm{s}} \sim g_{\mathrm{g}}^{4}$. In the present paper, we contrast these two hierarchy assumptions and illustrate the differences in the phenomenological predictions of minimal conformal extensions of the standard model.
\end{abstract}

DOI: 10.1103/PhysRevD.99.015026

\section{INTRODUCTION}

The standard model (SM) of particle physics represents a quantum field theory staying perturbatively consistent under renormalization group (RG) flow all the way up to the Planck scale $M_{\mathrm{Pl}}$, where quantum gravity effects become relevant. Due to the absence of any beyond-thestandard-model signals from the LHC, the conservative scenario of no (or minimal) new physics up to $M_{\mathrm{Pl}}$ has gained some momentum in the community. Still, there is a number of obvious shortcomings. For one, the Higgs mass parameter is unnaturally small compared to $M_{\mathrm{Pl}}$, known as the hierarchy problem. Moreover, the strong observational evidence for dark matter and neutrino masses calls for an extension of the SM. Ideally, such an extension should also resolve the puzzle of the metastable Higgs vacuum $[1,2]$.

A good guideline for extending the SM is to call for additional symmetries, such as supersymmetry, which should be broken in order to agree with current experimental observations. In this paper, we focus on another prominent example of a guiding (and broken) symmetry: The standard model is "nearly" conformal as a classical field theory. Conformal invariance is only broken by the explicit Higgs field mass term, which induces the electroweak symmetry breaking and the masses of all known

\footnotetext{
*loebbert@physik.hu-berlin.de †miczajka@physik.hu-berlin.de *plefka@physik.hu-berlin.de
}

Published by the American Physical Society under the terms of the Creative Commons Attribution 4.0 International license. Further distribution of this work must maintain attribution to the author(s) and the published article's title, journal citation, and DOI. Funded by SCOAP ${ }^{3}$. elementary particles. At least qualitatively, the same effect can be generated in a classically scale-free model, the radiative corrections of which lead to a spontaneous generation of mass scale and symmetry breaking as was first advocated by Coleman and Weinberg [3] (see Ref. [4] for an extensive review). While classical conformal symmetry is broken via radiative corrections, the vanishing mass term is stable under renormalization [5] using dimensional regularization. However, this attractive scenario does not yield a radiative breaking of the electroweak symmetry in a scalefree version of the SM (simply dropping the scalar mass term) due to the largeness of the top mass. By adding additional bosonic degrees of freedom (d.o.f.) to the SMe.g., via an extended Higgs sector or novel gauge fieldsthis problem may in principle be cured. The question for realizing such a (minimal) version of a conformal standard model has recently attracted a lot of attention in the literature; see e.g., [6-15]. A common feature of these works is an extended scalar sector for which an effective potential needs to be established and minimized such that spontaneously generated masses may be extracted in a perturbatively consistent and also gauge invariant fashion. As we shall argue in this paper, this is a nontrivial issue building upon certain scaling assumptions of the scalar couplings $\lambda_{i}$ with respect to the gauge and Yukawa couplings.

Besides the Coleman-Weinberg mechanism, another way to break conformal symmetry is via the introduction of a (typically high) cutoff scale $\Lambda$. This scale induces quadratic divergences generating a Higgs mass contribution proportional to $\Lambda^{2}$. In the context of conformal extensions of the standard model, this naturalness problem is an often addressed point of criticism (see e.g., Ref. [16]), since the classically vanishing Higgs mass requires fine-tuning in order to stay small. Here, we do not employ a cutoff scale as 
we only consider conformal extensions of the standard model designed such that Landau poles are delayed beyond the Planck scale and thus may be effectively considered as having no physical cutoff. We perform all calculations using dimensional regularization and employ the $\overline{\mathrm{MS}}$ renormalization scheme. We will also not address the question of embedding the considered models into a theory of quantum gravity which would result in a natural cutoff $\Lambda \sim M_{\mathrm{Pl}}$ at the Planck scale.

\section{A. Multiscale issue}

An immediate problem that one faces when considering multiscalar conformal extensions of the standard model is the question of multiscale renormalization. Loop contributions to the effective potential typically come with logarithms that depend on the ratio of the scalar field and the renormalization scale. These contributions become large when the field value and the scale differ significantly, which invalidates the perturbative expansion. In the case of a single scalar field, a single renormalization scale is sufficient to resum these logarithmic contributions via the RG which results in the RG-improved effective potential. What is done here is setting the arbitrary renormalization scale $\mu$ to the scalar field value at its minimum $\langle\phi\rangle$, which cancels all $\log$ arithms $\log \frac{\phi}{\langle\phi\rangle}$ in the potential and derived quantities thereof in the vacuum configuration. However, in the presence of multiple scalar fields, one faces logarithms of different field-to-scale ratios, which renders a single renormalization scale insufficient for dealing with all logarithmic contributions at the same time. A natural resolution seems to be the introduction of multiple renormalization scales as proposed by Einhorn and Jones [17] and later refined by Ford and Wiesendanger [18]. At least in certain cases, this approach was argued [19] to be equivalent to the decoupling method of Ref. [20], which splits the mutliscale problem into single-scale problems in between different mass thresholds. Unfortunately, these methods complicate the RG analysis significantly, which limits their applicability to simpler toy models or low loop orders. A more powerful approach was suggested by Gildener and Weinberg [21]: Assuming the presence of a flat direction of the classical potential in the space of scalar fields at some renormalization scale $\mu_{\mathrm{GW}}$, the symmetry breaking is studied only in this direction, which again results in a single-scale problem. In the context of conformal extensions of the standard model, this approach was recently applied in e.g., Ref. [14]. A new method for the study of multiscale potentials was suggested in Ref. [22], which assumes the existence of a (fielddependent) value of the renormalization scale where all loop corrections to the effective potential vanish. Working at this scale translates the problem of understanding the full effective potential into a study of the tree-level potential with running coupling constants.
Notably, even in a (single-scale) textbook approach, the problem of multiscale renormalization can be avoided in certain theories at one-loop order. This is due to a special prescription for solving the minimum conditions for the effective potential as illustrated in Sec. III for the model of Ref. [7].

\section{B. Gauge-dependence issue}

Besides the problem of multiscale renormalization, another important requirement is gauge independence. The effective potential is generically gauge dependent [23], and great care is needed to extract physical information contained in its minimal value. However, drawing conclusions on which modifications of the standard model are compatible with current experimental data is very sensitive to small modifications, and thus it requires caution to identify a minimal model. In particular, the gauge dependence of the effective potential has recently been emphasized in Refs. [24,25]. In the context of the standard model, gauge invariance was shown to require a nonstandard hierarchy of coupling constants [25], which amounts to taking the scalar coupling(s) $\lambda_{s}$ to be of the order of the fourth power of the gauge couplings $g_{i}$ and Yukawa couplings $y_{t}$, i.e.,

$$
\lambda_{s} \sim \mathcal{O}\left(g_{i}^{4}\right) \sim \mathcal{O}\left(y_{t}^{4}\right) .
$$

This hierarchy has also been shown to hold in the Coleman-Weinberg radiative symmetry breaking for scalar electrodynamics [3]. Reintroducing $\hbar$, this scaling is easily motivated by setting $\lambda_{s} \sim \hbar$; in other words, the classical scalar potential is made quantum by hand. Although being rather unconventional, this choice clearly allows the leading scalar potential to receive sizable oneloop quantum corrections that can significantly shift its minimum to nonvanishing field values $\langle\phi\rangle \neq 0$. As this scaling assumption makes the (tree-level) scalar potential essentially quantum, we term it the "quantum potential" approach.

The ordinary loop counting, on the other hand, amounts to the assumption

$$
\lambda_{s} \sim \mathcal{O}\left(g_{i}^{2}\right) \sim \mathcal{O}\left(y_{t}^{2}\right),
$$

which is equivalent to taking $\lambda_{s}$ not to be of order $\hbar$ as is usually done. In fact, this is the scaling applied in the Gildener-Weinberg scheme.

\section{Setup}

Let us explain the difference of the generic situation we are facing in multiscalar extensions of the conformal $\mathrm{SM}$ in more detail. Due to the assumed classical scale invariance, the tree-level part of the scalar potential needs to be of the form 


$$
V_{0}(\vec{\Phi})=\frac{1}{4} \lambda_{I J K L} \Phi_{I} \Phi_{J} \Phi_{K} \Phi_{L}
$$

where the totally symmetric symbol $\lambda_{I J K L}$ parametrizes the set of scalar couplings and the $\Phi_{I}(x)$ denote all scalar fields in the theory. The one-loop correction to the scalar potential computed in dimensional regularization then takes the generic form

$V_{\mathrm{eff}}^{(1)}=\frac{\hbar}{64 \pi^{2}} \sum_{i} n_{i} m_{i}\left[\Phi_{I}\right]^{4}\left(\ln \frac{m_{i}\left[\Phi_{I}\right]^{2}}{\mu^{2}}-a_{i}-\frac{1}{\epsilon}\right)$.

Here, the index $i$ runs over all particles in the theory which couple to the scalars. For each particle, $m_{i}\left[\Phi_{I}\right]$ denotes its field-dependent effective tree-level mass, which emerges for nonzero scalar vacuum expectation values and implicitly depends on the renormalization scale $\mu$. The $n_{i}$ count the real d.o.f. of the particle $i$ with a minus sign for fermions, while the $a_{i}$ are scheme-dependent constants: in the $\overline{\mathrm{MS}}$ scheme, they are given by $-5 / 6$ for gauge bosons and $-3 / 2$ for fermions or scalars. Clearly, the classical potential always has the trivial vacuum $\langle\vec{\Phi}\rangle=0$. The quest is now to have $V=V_{0}+V_{\text {eff }}^{(1)}$ develop a minimum at a nonzero value of $\langle\vec{\Phi}\rangle$ through radiative corrections. Here, we focus on the comparison of two distinct scenarios:

(1) Quantum-potential approach.-The multiscalar effective potential is treated as in the single-scalar case, e.g., in the case of the standard model. The scalar couplings $\lambda_{I J K L}$ are taken to scale as $\hbar$, i.e., to be of the same order of magnitude as (part of) the one-loop contributions $V_{\text {eff }}^{(1)}$ to the effective potential. Effectively, this amounts to assuming a hierarchy of couplings $\lambda \sim g^{4}$, where $g$ are the gauge or Yukawa couplings. This scaling hierarchy pushes the scalar coupling contributions in $V_{\mathrm{eff}}^{(1)}$ to the next order.

(2) Gildener-Weinberg method.-One demands that $V_{0}$ has a degenerate zero energy vacuum along a ray $\langle\Phi\rangle=\varphi \vec{n}$, parametrized by a sliding-scale field $\varphi$ at a particular scale $\mu_{\mathrm{GW}}$. The quantum fluctuations of $V_{\text {eff }}^{(1)}$ then lift the degeneracy along this valley and yield a radiatively generated nonvanishing vacuum expectation value $\langle\vec{\Phi}\rangle$, which induces all the masses in the theory. In this approach, the ordinary loop hierarchy of couplings $\lambda \sim g^{2}$ is assumed. Importantly, finding the minimum of the multiscalar effective potential reduces to a single scalar problem for the field $\varphi$.

In the case of single-scalar models, it can be seen that both scenarios yield the same result at one-loop order. This is due to the fact that for a single scalar potential $\lambda \phi^{4}$ the existence of a classically flat direction necessitates $\lambda\left(\mu_{\mathrm{GW}}\right)=0$ in the Gildener-Weinberg scenario. Then, $V_{\text {eff }}^{(1)}$ only depends on the gauge and Yukawa couplings of order $g^{4}$, just as is the case in the Coleman-Weinberg setup (at one-loop order). In the multiscalar case, however, radiative symmetry breaking is also possible if one assumes standard perturbative scalings, i.e., $\lambda \sim g^{2}$, since the now possible nontrivial vacuum degeneracy of the tree-level potential may be lifted by a small $\mathcal{O}(\hbar)$ one-loop correction. In the present paper, we contrast these two different scaling assumptions in the multiscalar case, i.e., the two different methods described above, in order to extract gauge invariant data from the effective potential. As we shall show, these scaling assumptions for the couplings lead to different phenomenological predictions in conformal extensions of the standard model, which have been very popular recently.

In the following analyses, we neglect influences of all leptons except for the top quark. Furthermore, since we work entirely at one-loop level and the Higgs boson has no color charge, we can neglect all contributions to the effective potential that come from the strong interaction. They will be of higher loop order.

The paper is structured as follows. We start by introducing the Hempfling model of Ref. [7] in Sec. II as our laboratory throughout the paper. We proceed to apply the quantum potential (QP) approach and the GildenerWeinberg (GW) method in Secs. III and IV, respectively. In particular, for both cases, we determine the allowed mass ranges for the new scalar and the new $\mathrm{U}(1)$ gauge boson $Z^{\prime}$, as well as the allowed couplings, demanding compatibility with experimental bounds on the scalar mixing and the absence of Landau poles and vacuum instability up to the Planck scale. In Sec. V, we briefly demonstrate that a further reduction of the field content does not lead to a phenomenologically viable model. Finally, we conclude by comparing the QP and GW approaches and give a brief outlook.

\section{OUR LABORATORY: THE HEMPFLING MODEL}

It is well known that implementing Coleman-Weinberg symmetry breaking into the standard model with vanishing mass term does not give rise to a phenomenologically viable vacuum due to the large top mass. As we will see below, the same applies to an extension of this model by a single scalar field; see also Ref. [14]. The addition of new fermionic d.o.f. will give negative contributions to the mass eigenvalues and only worsen the situation. We are thus led to introduce new bosonic fields. Restricting to renormalizable models, we can add scalar fields or vector fields.

\section{A. Hempfling model}

Here, we will analyze the conformal extension of the standard model that was proposed by Hempfling already in 1996, i.e., before the discovery of the Higgs boson. In addition to the standard model at vanishing tree-level Higgs mass, the Hempfling model contains a new "dark" U(1) 
gauge boson $Z_{\mu}^{\prime}$ exclusively coupled to a new scalar field $S$. This is a minimal extension of the conformal standard model in the following sense: As we will illustrate in Sec. V, an extension by a single scalar field $S$ alone is not capable of consistently reproducing the correct Higgs mass. Hence, we must add additional bosonic d.o.f. Adding yet another scalar would introduce several new couplings to the Higgs and the scalar $S$. We thus add a new Abelian gauge field $Z_{\mu}^{\prime}$ coupled only to the new scalar $S$ by the new gauge coupling $g_{Z^{\prime}}$. The complex scalar $S$ has a U(1) phase symmetry $S \rightarrow e^{i \alpha} S$, and the full Lagrangian for this model is given by

$$
\begin{aligned}
\mathcal{L}_{\mathrm{Hempf}}= & \left.\mathcal{L}_{\mathrm{SM}}\right|_{{ }_{m_{\mathrm{H}}=0}=0}-V(H, S)+\mathcal{D}_{\mu} S\left(\mathcal{D}^{\mu} S\right)^{\dagger}-\frac{1}{4} F_{\mu \nu}^{\prime} F^{\prime \mu \nu} \\
& +\mathcal{L}_{\mathrm{GF}}+\mathcal{L}_{\text {ghosts }},
\end{aligned}
$$

where $\left.\mathcal{L}_{\mathrm{SM}}\right|_{\substack{\lambda=0 \\ m_{\mathrm{H}}=0}}$ is the SM Lagrangian without the Higgs potential. The new tree-level potential is given by

$$
V(H, S)=\lambda_{1}\left(H^{\dagger} H\right)^{2}+\lambda_{12}\left(H^{\dagger} H\right)\left(S^{\dagger} S\right)+\lambda_{2}\left(S^{\dagger} S\right)^{2} .
$$

We work with the gauge fixing terms

$$
\mathcal{L}_{\mathrm{GF}}=-\frac{1}{2 \xi_{B}}\left(\partial_{\mu} B^{\mu}\right)^{2}-\frac{1}{2 \xi_{W}}\left(\partial_{\mu} A^{a \mu}\right)^{2}-\frac{1}{2 \xi_{Z^{\prime}}}\left(\partial_{\mu} Z^{\prime \mu}\right)^{2},
$$

keeping the $\xi_{i}$ arbitrary. Note that we also do not consider $U(1)$ mixing $F_{\mu \nu} F^{\prime \mu \nu}$ with the photon $A_{\mu}$. Next, the Higgs doublet and the new scalar are written in a background field $(\hat{\phi}, \hat{S}) \in \mathbb{R}$ expansion as
$H=\frac{1}{\sqrt{2}}\left(\begin{array}{c}\phi_{1}+i \psi_{1} \\ \hat{\phi}+\phi_{2}+i \psi_{2}\end{array}\right), \quad S=\frac{1}{\sqrt{2}}\left(\hat{S}+s_{1}+i s_{2}\right)$.

Terms involving ghosts or the strong interactions may be left out, as they only appear at higher loops. The covariant derivative couples $S$ to the new gauge field $Z^{\prime \mu}$ according to

$$
\mathcal{D}_{\mu} S=\left(\partial_{\mu}+i g_{Z^{\prime}} Z_{\mu}^{\prime}\right) S,
$$

inducing cubic and quartic interactions. Because the new gauge field does not interact directly with the fields of the standard model, we call it a dark gauge field.

\section{B. Effective potential}

As usual, we expand the scalar fields around classical field values $(\hat{\phi}, \hat{S})$ and integrate out the quantum fields to arrive at the effective potential at one-loop order. Dropping from now on the hats of the background fields, we have

$$
\begin{aligned}
V_{\text {eff }}(\phi, S)= & \frac{\lambda_{1}}{4} \phi^{4}+\frac{\lambda_{12}}{4} \phi^{2} S^{2}+\frac{\lambda_{2}}{4} S^{4} \\
& +\frac{1}{64 \pi^{2}} \sum_{i \in I} n_{i} m_{i}^{4}\left(\ln \frac{m_{i}^{2}}{\mu^{2}}-a_{i}-\frac{1}{\epsilon}\right),
\end{aligned}
$$

with $I=\left\{A, B, C, E_{ \pm}, F_{ \pm}, G_{ \pm}, I_{ \pm}, T\right\}$. The field-dependent masses are given by

$$
\begin{array}{ll}
m_{A}^{2}=\frac{g_{2}^{2}}{4} \phi^{2}, & m_{B}^{2}=\frac{\left(g_{1}^{2}+g_{2}^{2}\right)}{4} \phi^{2}, \\
m_{C}^{2}=g_{Z^{\prime}}^{2} S^{2}, & m_{T}^{2}=\frac{y_{t}^{2}}{2} \phi^{2},
\end{array}
$$

as well as

$$
\begin{aligned}
& m_{E \pm}^{2}=\frac{1}{4}\left(\left(6 \lambda_{1}+\lambda_{12}\right) \phi^{2}+\left(6 \lambda_{2}+\lambda_{12}\right) S^{2} \pm \sqrt{\left(\left(6 \lambda_{1}-\lambda_{12}\right) \phi^{2}-\left(6 \lambda_{2}-\lambda_{12}\right) S^{2}\right)^{2}+16 \lambda_{12}^{2} \phi^{2} S^{2}}\right) \\
& m_{F \pm}^{2}=\frac{1}{4}\left(2 \lambda_{2} S^{2}+\lambda_{12} \phi^{2} \pm \sqrt{\left(2 \lambda_{2} S^{2}+\lambda_{12} \phi^{2}\right)^{2}-\xi_{Z^{\prime}}\left(4 \lambda_{2} g_{Z^{\prime}}^{2} S^{4}+2 \lambda_{12} g_{Z^{\prime}}^{2} \phi^{2} S^{2}\right)}\right), \\
& m_{G \pm}^{2}=\frac{1}{4}\left(2 \lambda_{1} \phi^{2}+\lambda_{12} S^{2} \pm \sqrt{\left(2 \lambda_{1} \phi^{2}+\lambda_{12} S^{2}\right)^{2}-\left(\xi_{B} g_{1}^{2}+\xi_{W} g_{2}^{2}\right)\left(4 \lambda_{1} \phi^{4}+2 \lambda_{12} \phi^{2} S^{2}\right)}\right), \\
& m_{I \pm}^{2}=\frac{1}{4}\left(2 \lambda_{1} \phi^{2}+\lambda_{12} S^{2} \pm \sqrt{\left(2 \lambda_{1} \phi^{2}+\lambda_{12} S^{2}\right)^{2}-\xi_{W} g_{2}^{2}\left(4 \lambda_{1} \phi^{4}+2 \lambda_{12} \phi^{2} S^{2}\right)}\right),
\end{aligned}
$$

while the parameters $n_{i}$ and $a_{i}$ take the form

$$
\begin{aligned}
& n_{A}=6, \quad n_{B}=3, \quad n_{C}=3, \quad n_{T}=-12, \quad n_{E}=n_{F}=n_{G}=1=\frac{1}{2} n_{I}, \\
& a_{A}=a_{B}=a_{C}=-\frac{5}{6}, \quad a_{E}=a_{F}=a_{G}=a_{I}=a_{T}=-\frac{3}{2} .
\end{aligned}
$$

Note that of all leptons only the top quark is included in the analysis, as its couplings $y_{t}$ is by far dominant. 


\section{Beta functions}

The one-loop beta functions for the Hempfling model are given by $[14,26]$

$$
\begin{aligned}
\beta_{\lambda_{1}}= & 24 \lambda_{1}^{2}+\lambda_{12}^{2}-3 \lambda_{1}\left(g_{1}^{2}+3 g_{2}^{2}\right)+\frac{3}{8}\left(g_{1}^{4}+2 g_{1}^{2} g_{2}^{2}+3 g_{2}^{4}\right) \\
& +12 \lambda_{1} y_{t}^{2}-6 y_{t}^{4}, \\
\beta_{\lambda_{12}}= & \lambda_{12}\left(12 \lambda_{1}+8 \lambda_{2}+4 \lambda_{12}+6 y_{t}^{2}-\frac{3}{2}\left(g_{1}^{2}+3 g_{2}^{2}\right)-6 g_{Z^{\prime}}^{2}\right), \\
\beta_{\lambda_{2}}= & 20 \lambda_{2}^{2}+2 \lambda_{12}^{2}-6 \lambda_{2} g_{Z^{\prime}}^{2}+6 g_{Z^{\prime}}^{4} \\
\beta_{g_{1}}= & \frac{41}{6} g_{1}^{3}, \quad \beta_{g_{2}}=-\frac{19}{6} g_{2}^{3}, \quad \beta_{g_{Z^{\prime}}}=\frac{1}{3} g_{Z^{\prime}}^{3}, \\
\beta_{g_{3}}= & -7 g_{3}^{3}, \quad \beta_{y_{t}}=y_{t}\left(\frac{9}{2} y_{t}^{2}-\frac{17}{12} g_{1}^{2}-\frac{9}{4} g_{2}^{2}-8 g_{3}^{2}\right),
\end{aligned}
$$

where $\beta_{\alpha}=16 \pi^{2} \mu \frac{\mathrm{d} \alpha}{\mathrm{d} \mu}$.

\section{QUANTUM POTENTIAL APPROACH}

In this section, we consider the quantum potential method and apply it to the concrete example of the Hempfling model. We discuss the phenomenological consistency with the observed value of the Higgs mass and study the absence of Landau poles and vacuum stability up to the Planck scale.

\section{A. Conceptual idea}

As motivated in the Introduction, we will now impose the hierarchy of coupling constants

$$
\lambda_{j} \sim g_{k}^{4} \sim y_{t}^{4} \sim \hbar,
$$

which allows us to consistently expand all quantities in the small parameter $\hbar$. This assumption extrapolates the hierarchy $\lambda \sim g^{4}$ of scalar electrodynamics which-in that model —was explicitly proven by Coleman and Weinberg using the renormalization group [3]. Indeed, for theories with a single scalar field, this hierarchy of couplings is necessary in order for a one-loop contribution to push the minimum of the tree-level potential from zero field values to a nonzero value, i.e., to implement Coleman-Weinberg symmetry breaking. Moreover, this hierarchy is crucial to guarantee the gauge independence of physical information extracted from the Coleman-Weinberg potential [24]. Also for the standard model, imposing the hierarchy (3.1) consistently guarantees trustable and gauge-independent results [25].

\section{B. QP of Hempfling model}

Solving the stationarity conditions

$$
0=\left.\frac{\mathrm{d} V_{\text {eff }}}{\mathrm{d} \phi}\right|_{\phi=\langle\phi\rangle, S=\langle S\rangle}, \quad 0=\left.\frac{\mathrm{d} V_{\text {eff }}}{\mathrm{d} S}\right|_{\phi=\langle\phi\rangle, S=\langle S\rangle},
$$

for $\lambda_{1}$ and $\lambda_{2}$, we arrive at the one-loop effective potential

$$
\begin{aligned}
V_{\mathrm{eff}}^{\mathrm{ren}}(\phi, S)= & -\frac{\lambda_{12}}{8}\langle\phi\rangle^{2}\langle S\rangle^{2}\left(\frac{\phi^{2}}{\langle\phi\rangle^{2}}-\frac{S^{2}}{\langle S\rangle^{2}}\right)^{2} \\
& +\frac{3}{64 \pi^{2}} g_{Z^{\prime}}^{4} S^{4}\left(\log \frac{S^{2}}{\langle S\rangle^{2}}-\frac{1}{2}\right) \\
& +\frac{3}{64 \pi^{2}} \frac{\phi^{4}}{\langle\phi\rangle^{4}}\left(2 m_{W}^{4}+m_{Z}^{4}-4 m_{t}^{4}\right) \\
& \times\left(\log \frac{\phi^{2}}{\langle\phi\rangle^{2}}-\frac{1}{2}\right),
\end{aligned}
$$

where we have

$m_{W}=\frac{g_{2}}{2}\langle\phi\rangle, \quad m_{Z}=\frac{\sqrt{g_{1}^{2}+g_{2}^{2}}}{2}\langle\phi\rangle, \quad m_{t}=\frac{y_{t}}{\sqrt{2}}\langle\phi\rangle$.

Crucially, all dependence on the gauge parameters disappears from the effective potential (3.3) after imposing the hierarchy of couplings. That is, as in the standard model, the hierarchy (3.1) resolves the gauge-dependence issue. Moreover, solving (3.2) symmetrically for $\lambda_{1}$ and $\lambda_{2}$ (instead of e.g., for $\lambda_{1}$ and $\lambda_{12}$ ), the dependence of the logarithms on the renormalization scale $\mu$ completely drops out of the effective potential. In particular, we have no multiscale problem which naively could have been expected, cf. the Introduction. This simplifies the analysis considerably. This property is a consequence of the vanishing of $\beta_{\lambda_{12}}$ at one loop under the scaling assumptions $\lambda_{i} \sim \mathcal{O}\left(g_{i}^{4}\right)$; compare (2.10). We do not expect it to prevail at higher loop orders.

The above effective potential has a minimum at $\{\langle\phi\rangle,\langle S\rangle\}$. Since the Higgs vacuum expectation value is fixed by experiment, the potential contains three free parameters, namely $\left\{\lambda_{12}, g_{Z^{\prime}},\langle S\rangle\right\}$. However, we can fix an additional one by demanding the existence of a mass eigenstate with eigenvalue $m_{h}$, the measured Higgs mass.

In order to do so, we calculate the Hessian of the potential at its minimum

$$
\begin{aligned}
M_{i j}^{2} & =\left.\frac{\partial^{2} V_{\text {eff }}^{\mathrm{ren}}}{\partial \phi_{i} \partial \phi_{j}}\right|_{\phi=\langle\phi\rangle, S=\langle S\rangle} \\
& =\left(\begin{array}{cc}
-\frac{m_{0}^{4}}{8 \pi^{2}\langle\phi\rangle^{2}}-\lambda_{12}\langle S\rangle^{2} & \lambda_{12}\langle S\rangle\langle\phi\rangle \\
\lambda_{12}\langle S\rangle\langle\phi\rangle & \frac{3 g_{Z^{\prime}}^{4}\langle S\rangle^{2}}{8 \pi^{2}}-\lambda_{12}\langle\phi\rangle^{2}
\end{array}\right),
\end{aligned}
$$


where we have introduced the abbreviation

$$
m_{0}^{4}=12 m_{t}^{4}-6 m_{W}^{4}-3 m_{Z}^{4}=(319 \mathrm{GeV})^{4} .
$$

Since this matrix is nondiagonal, the mass eigenstates will consist of mixtures of the interaction eigenstates. It is of course simple to write down analytic expressions for the mass eigenvalues

$$
m_{ \pm}^{2}\left(\lambda_{12}, g_{Z^{\prime}},\langle S\rangle\right)=\frac{1}{2} \operatorname{tr} M^{2} \pm \sqrt{\left(\frac{\operatorname{tr} M^{2}}{2}\right)^{2}-\operatorname{det} M^{2}}
$$

but it is not possible to invert them in a closed form for any of the three free parameters. In order to match one of them to the Higgs mass, we will therefore numerically solve the resulting equation.

Now, the presence of two mass eigenvalues opens up two possible scenarios: The Higgs may be lighter or heavier than the new scalar $m_{X}$, i.e., $m_{h}=m_{ \pm}$and $m_{X}=m_{\mp}$. We term these scenarios A $\left(m_{h}=m_{-}\right)$and $\mathrm{B}\left(m_{h}=m_{+}\right)$. Nevertheless, the numerical procedure is straightforward in both cases; we randomly dial $3 \times 10^{4}$ pairs $\lambda_{12} \in[0,-\pi]$ and $g_{Z^{\prime}} \in[0, \pi]$ then:

(1) Solve the equation

$$
m_{ \pm}\left(\lambda_{12}, g_{Z^{\prime}},\langle S\rangle\right)=m_{h}
$$

for the expectation value $\langle S\rangle\left(\lambda_{12}, g_{Z^{\prime}}\right)$.

(2) Eliminate $\langle S\rangle$ in the other mass eigenvalue, resulting in

$$
m_{\mp}\left(\lambda_{12}, g_{Z^{\prime}}\right)=m_{\mp}\left(\lambda_{12}, g_{Z^{\prime}},\langle S\rangle\left(\lambda_{12}, g_{Z^{\prime}}\right)\right) .
$$

(3) For any given pair $\left\{\lambda_{12}, g_{Z^{\prime}}\right\}$ compute the couplings $\left\{\lambda_{1}, \lambda_{2}\right\}$ from (3.2) as well as the predicted masses for the new d.o.f.

$$
m_{X}=m_{\mp}\left(\lambda_{12}, g_{Z^{\prime}}\right), \quad m_{Z^{\prime}}=g_{Z^{\prime}}\langle S\rangle\left(\lambda_{12}, g_{Z^{\prime}}\right),
$$

which can be used as initial conditions for the RG equations.

By constructing the appropriate parameter regions, we enforce perturbativity by demanding $\left|\lambda_{i}\right|<\pi$. We also immediately dismiss parameter sets, which lead to unstable extrema of the effective potential (i.e., negative values for one of the $m_{i}^{2}$ ) or to sizeable mixing between the Higgs and the new scalar:

$$
m_{ \pm}=m_{h}=\cos \alpha \phi+\sin \alpha S .
$$

Following the analysis of Refs. [12,27], this mixing is constrained to $\sin \alpha<0.44$ from present experimental bounds. In addition, we study the UV consistency of the model: We integrate the beta functions (enforcing the scaling assumption) up to the scale at which a Landau pole or vacuum instability occurs and stop at $M_{\text {Planck }}$ if this does not occur.

We show a plot of scenario A in Fig. 1, in which the Higgs is lighter than the new scalar; there is a small window of allowed couplings which allows for an extrapolation all the way to the Planck scale. This window translates to a range of masses

$$
\begin{aligned}
& 650 \mathrm{GeV}<m_{Z^{\prime}}<970 \mathrm{GeV} \\
& 160 \mathrm{GeV}<m_{X}<250 \mathrm{GeV}
\end{aligned}
$$

for the dark $Z^{\prime}$ boson and the new scalar resonance; see Fig. 2. As can be seen from the plots of Fig. 3, scenario B taking the new scalar to be lighter than the Higgs does not allow for an extrapolation up to the Planck scale for any coupling.

\section{GILDENER-WEINBERG APPROACH}

Here, we study the effective potential of the Hempfling model using the Gildener-Weinberg method of Ref. [21].

\section{A. Conceptual idea}

In their classic quantum field theory paper, Gildener and Weinberg introduced an elegant formalism to deal with the perturbative construction of the effective potential in the presence of multiple scalar vacuum expectation values in classically scale invariant theories. It represents a generalization of the Coleman-Weinberg idea to the multiscalar case and is equivalent to it in the single field case. The key assumption is that the classical potential

$$
V_{0}(\vec{\Phi})=\frac{1}{4} \lambda_{I J K L} \Phi_{I} \Phi_{J} \Phi_{K} \Phi_{L}
$$

has a nontrivial minimum at nonzero field values $\langle\vec{\Phi}\rangle \neq \overrightarrow{0}$ at a particular scale $\mu_{\mathrm{GW}}$, the Gildener-Weinberg scale. This yields certain relations termed $R$ among the couplings $\lambda_{I J K L}$,

$$
\left.\frac{\partial V_{0}}{\partial \Phi_{i}}\right|_{\mu=\mu_{\mathrm{GW}},\langle\vec{\Phi}\rangle \neq 0}=\left.0 \Rightarrow R\left(\lambda_{I J K L}\right)\right|_{\mu=\mu_{\mathrm{GW}}}=0 .
$$

Due to scale invariance of $V_{0}(\vec{\Phi})$, this immediately implies that one has a vacuum degeneracy of $V_{0}$ along a ray going through the origin in scalar field space where the minimal value of the classical potential is zero,

$$
\langle\vec{\Phi}\rangle=\varphi \vec{n}, \quad V_{0}(\langle\vec{\Phi}\rangle)=0 .
$$

Here, $\varphi$ parametrizes the sliding scale, and we normalize $\vec{n}^{2}=1$. 

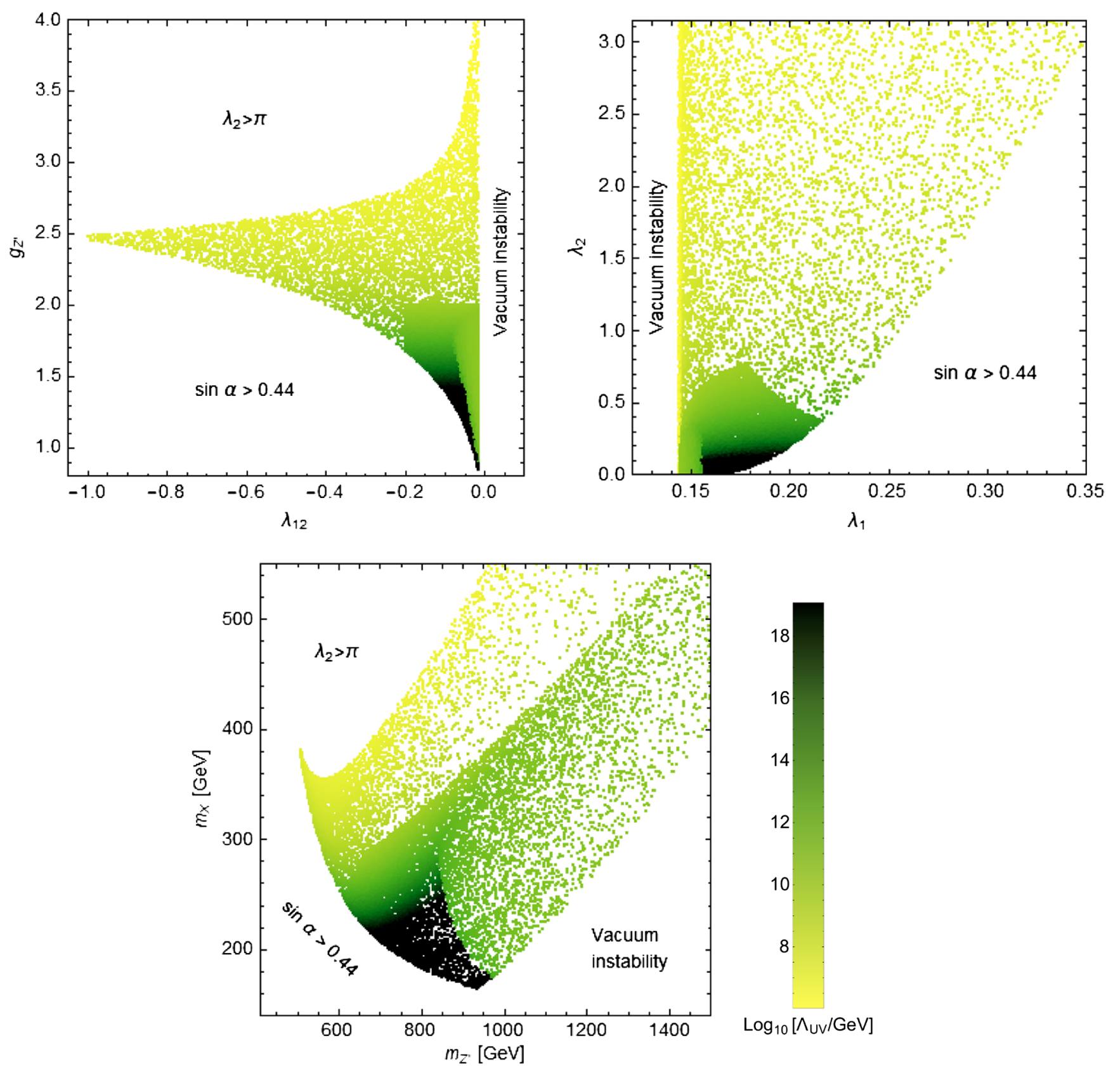

FIG. 1. Largest possible UV scales in the QP scenario A of the Hempfling model where the Higgs is the lighter scalar particle.

This vacuum degeneracy of the classical potential may be lifted by quantum fluctuations. Evaluating the one-loop contribution to the renormalized effective potential along the degenerate vacuum ray, one has

$$
V_{\mathrm{eff}}^{(1)}(\vec{\Phi}=\varphi \vec{n})=A \varphi^{4}+B \varphi^{4} \ln \frac{\varphi^{2}}{\mu_{\mathrm{GW}}^{2}} .
$$

Here, the functions $A$ and $B$ take the form

$$
\begin{aligned}
& A=\frac{\hbar}{64 \pi^{2}\langle\varphi\rangle^{4}} \sum_{i} n_{i} m_{i}[\langle\varphi\rangle \vec{n}]^{4}\left(\ln \frac{m_{i}[\langle\varphi\rangle \vec{n}]^{2}}{\langle\varphi\rangle^{2}}-a_{i}\right)=\frac{\hbar}{64 \pi^{2}} \sum_{i} n_{i} \tilde{m}_{i}[\vec{n}]^{4}\left(\ln \tilde{m}_{i}[\vec{n}]^{2}-a_{i}\right), \\
& B=\frac{\hbar}{64 \pi^{2}\langle\varphi\rangle^{4}} \sum_{i} n_{i} m_{i}[\langle\varphi\rangle \vec{n}]^{4}=\frac{\hbar}{64 \pi^{2}} \sum_{i} n_{i} \tilde{m}_{i}[\vec{n}]^{4} .
\end{aligned}
$$




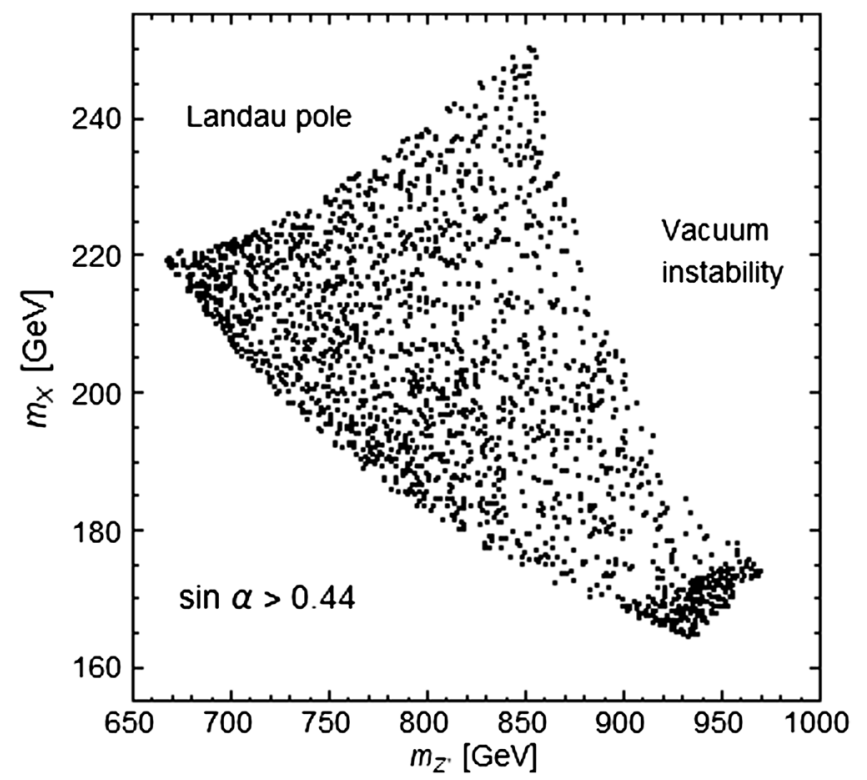

FIG. 2. Allowed mass ranges for the new scalar and Z' particles in the quantum potential scenario $\mathrm{A}\left(m_{X}>m_{h}\right)$ which are perturbatively stable up to the Planck scale. The scenario B $\left(m_{X}<m_{h}\right)$ always breaks down before reaching the Planck scale.

The vacuum expectation value $\langle\varphi\rangle$ for the sliding scale field is radiatively generated, and we generically have $m_{i}[\langle\varphi\rangle \vec{n}]=\langle\varphi\rangle \tilde{m}_{i}[\vec{n}]$ such that $A$ and $B$ are in fact independent of $\langle\varphi\rangle$ and are pure functions of the couplings. The extremum of the one-loop effective potential along the ray then lies at

$$
\frac{\langle\varphi\rangle}{\mu_{\mathrm{GW}}}=\exp \left[-\frac{1}{4}-\frac{A}{2 B}\right]
$$

Hence, as long as $A$ and $B$ are of the same order of magnitude, the logarithm $\ln \frac{\langle\varphi\rangle}{\mu_{\mathrm{GW}}}$ in the effective potential stays small, and the perturbative expansion is under control. One also straightforwardly extracts the mass of the excitation along the flat direction $\vec{n}$ which is the pseudo-Goldstone boson (PGB) of broken scale invariance. Originally massless, its mass is spontaneously generated by quantum fluctuations and given by the compact expression

$$
m_{\mathrm{PGB}}^{2}=\left.\frac{\mathrm{d}^{2} V_{\mathrm{eff}}^{(1)}(\varphi \vec{n})}{\mathrm{d} \varphi^{2}}\right|_{\varphi=\langle\varphi\rangle}=8 B\langle\varphi\rangle^{2}
$$

at one-loop precision. Clearly, a positive $B$ is required in order to have a minimum of the potential. In conformal extensions of the standard model, $m_{\mathrm{PGB}}$ may or may not be identified with the Higgs mass.

\section{B. GW for Hempfling model}

Let us now analyze the Hempfling model in the Gildener-Weinberg approach. The classical potential for the two real scalars $\phi$ and $S$ reads

$$
V_{0}(\vec{\Phi})=\frac{\lambda_{1}}{4} \phi^{4}+\frac{\lambda_{12}}{4} \phi^{2} S^{2}+\frac{\lambda_{2}}{4} S^{4}, \quad\left(\lambda_{1}, \lambda_{2}>0\right),
$$

where the positivity constraint on the scalar couplings implies stability. A degenerate nontrivial vacuum occurs if the condition

$$
\lambda_{12}=-2 \sqrt{\lambda_{1} \lambda_{2}}
$$

is met at $\mu=\mu_{\mathrm{GW}}$. At this scale, the classical potential takes the simple perfect square form

$$
V_{0}=\left(\frac{\sqrt{\lambda_{1}}}{2} \phi^{2}-\frac{\sqrt{\lambda_{2}}}{2} S^{2}\right)^{2}
$$

Clearly, we then have a degenerate vacuum along the ray

$$
\begin{aligned}
\left(\begin{array}{c}
\phi \\
S
\end{array}\right)_{\text {ray }} & =\varphi \vec{n}=\varphi\left(\begin{array}{c}
\cos \alpha \\
\sin \alpha
\end{array}\right) \\
& =\frac{\varphi}{\sqrt{\lambda_{1}^{1 / 2}+\lambda_{2}^{1 / 2}}}\left(\begin{array}{c}
\lambda_{2}^{1 / 4} \\
\lambda_{1}^{1 / 4}
\end{array}\right), \quad \text { with } \\
\tan \alpha & =\left(\frac{\lambda_{1}}{\lambda_{2}}\right)^{1 / 4} \cdot
\end{aligned}
$$

The particular form of the one-loop functions $A$ and $B$ in (4.4) may be straightforwardly read off from the results in (2.6) and (2.8). Remarkably, one finds that all the gauge parameter-dependent masses $m_{F \pm}, m_{G \pm}$, and $m_{I \pm}$ vanish identically on the ray (4.10) upon imposing the relation (4.9). ${ }^{1}$ Hence, in the GW setup with scaling assumptions $\lambda_{s} \sim \mathcal{O}\left(g_{i}^{2}\right) \sim \mathcal{O}\left(y_{t}^{2}\right)$, we do find explicit gauge invariance at leading order in perturbation theory. In addition, $m_{E-}$ vanishes as it corresponds to the tree-level mass of the pseudo-Goldstone boson excitation along the vacuum ray. For the nonvanishing dimensionless mass coefficients $\tilde{m}_{i}$, one then finds [enforcing the conditions (4.9) and (4.10)]

$$
\begin{aligned}
\tilde{m}_{A}^{2} & =\frac{g_{2}^{2} \sqrt{\lambda_{2}}}{4\left(\sqrt{\lambda_{1}}+\sqrt{\lambda_{2}}\right)}, \quad \tilde{m}_{B}^{2}=\frac{\left(g_{1}^{2}+g_{2}^{2}\right) \sqrt{\lambda_{2}}}{4\left(\sqrt{\lambda_{1}}+\sqrt{\lambda_{2}}\right)}, \\
\tilde{m}_{C}^{2} & =\frac{g_{Z^{\prime}}^{2} \sqrt{\lambda_{1}}}{\left(\sqrt{\lambda_{1}}+\sqrt{\lambda_{2}}\right)}, \\
\tilde{m}_{E+}^{2} & =2 \sqrt{\lambda_{1} \lambda_{2}}, \quad \tilde{m}_{T}^{2}=\frac{y_{t}^{2} \sqrt{\lambda_{2}}}{2\left(\sqrt{\lambda_{1}}+\sqrt{\lambda_{2}}\right)} .
\end{aligned}
$$

These are to be inserted into the definitions of the functions $A$ and $B$ in (4.5). The corresponding masses $m_{i}$ of the $W$ and $Z$ boson, the dark $Z^{\prime}$ boson, the scalar $\varphi_{E}$ as well as the

\footnotetext{
${ }^{1}$ In a related model, the gauge invariance in the GW approach was also noted in the Appendix of Ref. [10].
} 

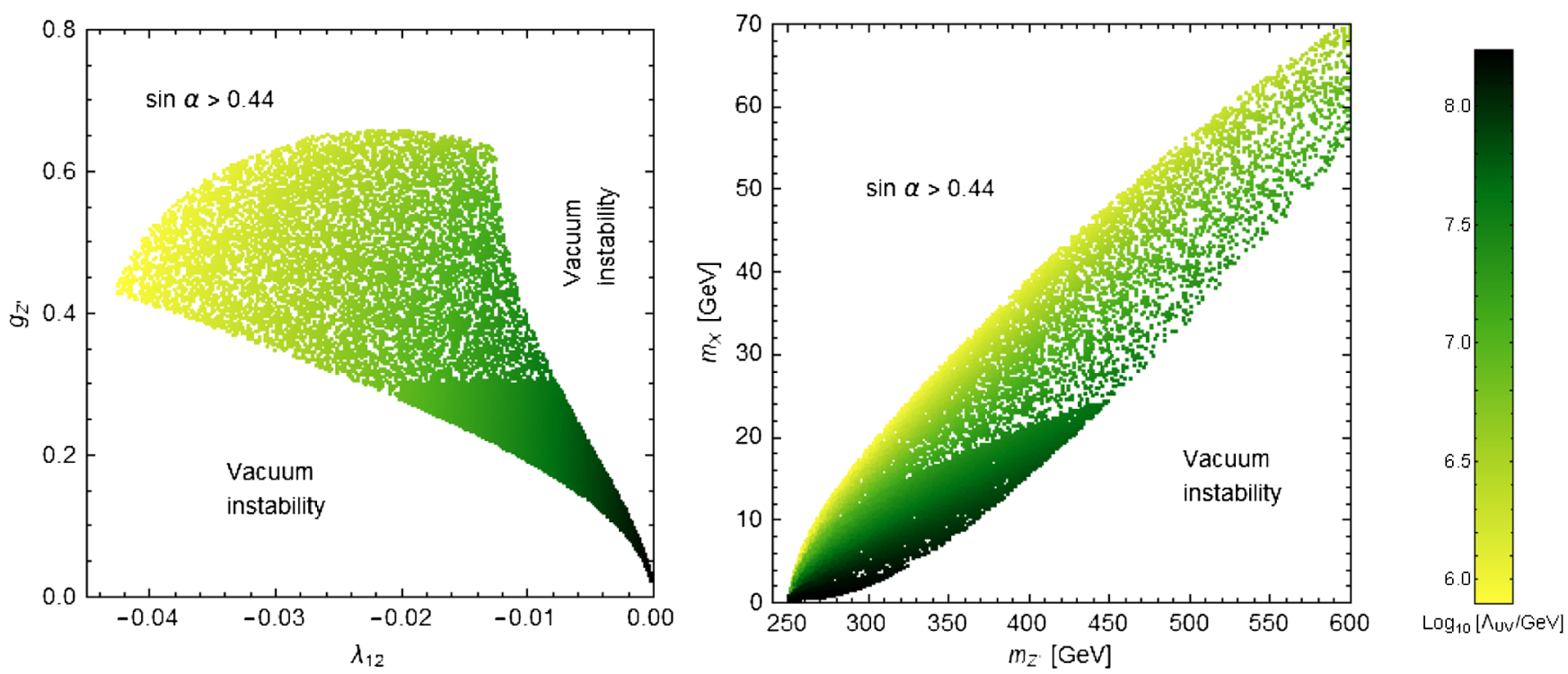

FIG. 3. Largest possible UV scales in the QP scenario B of the Hempfling model where the Higgs is the heavier scalar particle.

top quark $t$ are then obtained by multiplying these expressions by the vacuum expectation value of the sliding scale $\langle\varphi\rangle$, to wit

$$
\begin{array}{lll}
m_{W}=\tilde{m}_{A}\langle\varphi\rangle, & m_{Z}=\tilde{m}_{B}\langle\varphi\rangle, \quad m_{t}=\tilde{m}_{T}\langle\varphi\rangle, \\
m_{Z^{\prime}}=\tilde{m}_{C}\langle\varphi\rangle, & m_{E}=\tilde{m}_{E+}\langle\varphi\rangle .
\end{array}
$$

The mass of the pseudo-Goldstone boson then follows from the above and (4.7) to be

$$
m_{\mathrm{PGB}}^{2}=\frac{6 m_{W}^{4}+3 m_{Z}^{4}-12 m_{t}^{4}+3 m_{Z^{\prime}}^{4}+m_{E}^{4}}{8 \pi^{2}\langle\varphi\rangle^{2}}
$$

The vacuum expectation value $\langle\varphi\rangle$ is related to the vacuum expectation value of the SM-Higgs field $\langle\phi\rangle=246 \mathrm{GeV}$ via

$$
\langle\varphi\rangle=\frac{\sqrt{\lambda_{1}^{1 / 2}+\lambda_{2}^{1 / 2}}}{\lambda_{2}^{1 / 4}}\langle\phi\rangle .
$$

This relation then determines the masses of the dark $Z^{\prime}$ boson and second scalar $\varphi_{E}$ as functions of $\lambda_{1}$ and $\lambda_{2}$ to be

$m_{Z^{\prime}}=g_{Z^{\prime}}\left(\frac{\lambda_{1}}{\lambda_{2}}\right)^{1 / 4}\langle\phi\rangle, \quad m_{E}=\sqrt{2} \lambda_{1}^{1 / 4} \sqrt{\lambda_{1}^{1 / 2}+\lambda_{2}^{1 / 2}}\langle\phi\rangle$.

Moreover, the relation (4.6) determines the GildenerWeinberg scale $\mu_{\mathrm{GW}}$ as a function of $\lambda_{1}$ and $\lambda_{2}$ and the SM parameters:

$$
\mu_{\mathrm{GW}}=\exp \left[\frac{1}{4}+\frac{A}{2 B}\right] \frac{\sqrt{\lambda_{1}^{1 / 2}+\lambda_{2}^{1 / 2}}}{\lambda_{2}^{1 / 4}}\langle\phi\rangle .
$$

All unknown quantities have now been expressed as functions of $\lambda_{1}$ and $\lambda_{2}$. Note that all couplings here are defined at the scale $\mu_{\mathrm{GW}}$. This means that the SM quantities need in principle to be RG evolved from the electroweak scale to $\mu_{\mathrm{EW}}$. However, as long as the relative factor in (4.16) is not too different from 1 , this effect may be neglected.

Let us now look at the classical mass matrix in detail. One easily computes

$$
\begin{aligned}
M_{i j}^{2} & =\left.\frac{\partial^{2} V_{0}}{\partial \phi_{i} \partial \phi_{j}}\right|_{\phi=\langle\phi\rangle, S=\langle S\rangle} \\
& =\frac{2\langle\varphi\rangle^{2}}{\sqrt{\lambda_{1}}+\sqrt{\lambda_{2}}}\left(\begin{array}{cc}
\lambda_{1} \sqrt{\lambda_{2}} & -\left(\lambda_{1} \lambda_{2}\right)^{3 / 4} \\
-\left(\lambda_{1} \lambda_{2}\right)^{3 / 4} & \lambda_{2} \sqrt{\lambda_{1}}
\end{array}\right) .
\end{aligned}
$$

The two eigenstates corresponding to the scalar masses $m_{E}$ and $m_{\mathrm{PGB}}$ are expressed in terms of the initial scalar fields $\phi$ and $S$ as

$$
\begin{aligned}
\varphi_{\mathrm{PGB}} & =\cos \alpha \phi+\sin \alpha S, \\
\varphi_{E} & =-\sin \alpha \phi+\cos \alpha S, \\
\tan \alpha & =\left(\frac{\lambda_{1}}{\lambda_{2}}\right)^{1 / 4} .
\end{aligned}
$$

Importantly, there are now two options to identify the Higgs mass of $m_{h}=125 \mathrm{GeV}$ with the scalar resonances found: either $m_{h}=m_{\mathrm{PGB}}$ (scenario A) or $m_{h}=m_{E}$ (scenario B). Either choice determines yet another coupling such that in the end all quantities depend on just two parameters. 
As discussed in the previous section, present observational bounds at the LHC restrict the allowed mixing in the extended Higgs sector. The analyses of Refs. [12,27] restricted the mixing angle $\omega$ in the parametrization (translated to our conventions)

$$
h=\cos \omega \phi-\sin \omega S
$$

to $|\sin \omega|<0.44$, where $h$ is the Higgs field mass eigenstate. This translates to the mixing angle bounds $|\sin \alpha|<0.44$ in scenario A and $|\sin \alpha|>0.90$ in scenario B in (4.18).

\section{Phenomenological analysis}

The key equations are

$$
\begin{gathered}
3 m_{Z^{\prime}}^{4}+m_{E}^{4}=8 \pi^{2} m_{\mathrm{PGB}}^{2} \frac{\sqrt{\lambda_{1}}+\sqrt{\lambda_{2}}}{\sqrt{\lambda_{2}}}\langle\phi\rangle^{2}+m_{0}^{4} \\
m_{Z^{\prime}}=g_{Z^{\prime}}\left(\frac{\lambda_{1}}{\lambda_{2}}\right)^{1 / 4}\langle\phi\rangle \\
m_{E}=\sqrt{2} \lambda_{1}^{1 / 4}\left(\sqrt{\lambda_{1}}+\sqrt{\lambda_{2}}\right)^{1 / 2}\langle\phi\rangle,
\end{gathered}
$$

where $m_{0}^{4}=12 m_{t}^{4}-6 m_{W}^{2}-3 m_{Z}^{4}=(319 \mathrm{GeV})^{4}$ and $\langle\phi\rangle=$ $246 \mathrm{GeV}$. It is then clear that the mass of the $Z^{\prime}$ boson and the mass of the non-Higgs scalar will depend on a twoparameter family. In scenario $\mathrm{A}$, we will take $\left\{\lambda_{1}, \lambda_{2}\right\}$, while in scenario $\mathrm{B}$, we take $\left\{\lambda_{1}, g_{Z^{\prime}}\right\}$ as independent quantities. We randomly generate values for these couplings and check their perturbative validity by demanding the following bounds,

$$
\langle\varphi\rangle, \mu_{\mathrm{GW}} \in[24.6 \mathrm{GeV}, 2460 \mathrm{GeV}] ;
$$

i.e., these scales are only a factor of 10 away from the electroweak scale. Beyond this, we would have to RG evolve the SM parameters to $\mu_{\mathrm{GW}}$ consistently, which we did not implement in this work. Moreover, $\langle\varphi\rangle$ and $\mu_{\mathrm{GW}}$ are also allowed to differ by a factor of 10 in order to avoid large logarithms. Finally, we constrain the couplings $\left|\lambda_{i}\right|$ and $\left|g_{Z^{\prime}}\right|$ to be numerically smaller than $\pi$.

For every pair $\left\{\lambda_{1}, \lambda_{2}\right\}$ respectively $\left\{\lambda_{1}, g_{Z^{\prime}}\right\}$ the UVbreakdown scale is computed by integrating the RG equations using the initial conditions spelled out in the Appendix. A breakdown is quantified by any gauge coupling becoming larger than 10 (Landau pole) or the scalar couplings $\lambda_{1}$ or $\lambda_{2}$ turning negative (vacuum instability). For this, the one-loop RG equations of the Hempfling model were solved numerically, and the breakdown scale $\Lambda_{\mathrm{UV}}$ was recorded for every data point. We analyzed $\mathcal{O}\left(10^{4}\right)$ random points in both scenarios:

(i) Scenario $\mathrm{A}\left(m_{h}=m_{\mathrm{PGB}}\right)$ :

Here, we dial a pair $\left\{\lambda_{1}, \lambda_{2}\right\}$ of couplings to find $m_{E}$ from (4.22). Inserting this into (4.20) yields $m_{Z^{\prime}}$, and using this in (4.21) finally gives us $g_{Z^{\prime}}$.
The values of $\left\{\lambda_{1}, \lambda_{2}\right\}$ were picked randomly in the interval $[0, \pi]^{2}$. However, it turns out that the mixing condition $|\sin \alpha<0.44|$ is violated for all perturbatively viable resulting pairs $\left\{\lambda_{1}, \lambda_{2}\right\}$ in this scenario. Hence, this model is ruled out by experiment.

(ii) Scenario B $\left(m_{h}=m_{E}\right)$ :

Now, $\lambda_{1}$ and $\lambda_{2}$ are not independent. We therefore dial a pair $\left\{g_{Z^{\prime}}, \lambda_{1}\right\}$ within $[0, \pi]^{2}$. The coupling $\lambda_{2}$ then follows from solving (4.21) to be given by

$$
\lambda_{2}=\left(\frac{m_{h}^{2}}{2 \sqrt{\lambda_{1}}\langle\phi\rangle^{2}}-\sqrt{\lambda_{1}}\right)^{2} .
$$

Implementing the mixing constraint $\tan \alpha>2.04$, which amounts to $\lambda_{2}<\lambda_{1} / 2.04^{4}$, yields a very narrow range for $\lambda_{1}$ :

$$
\lambda_{1} \in[0.104,0.170] .
$$

This forces $\lambda_{2}$ to be very small, $\lambda_{2}<0.01$ and accordingly $\left|\lambda_{12}\right|<0.08$. Then, the mass of the $Z^{\prime}$ boson follows directly from (4.21), and the mass of the non-Higgs scalar $m_{\mathrm{PGB}}$ is deduced from (4.20). After checking the RG evolution of all couplings, we plot the breakdown scale in heat plots of Fig. 4. One sees that also the gauge coupling is narrowed down by the UV conditions to $g_{Z^{\prime}}<0.9$. Above this value, it develops a Landau pole before $M_{\mathrm{Pl}}$ is reached. Note that the SM value $\lambda_{1}=m_{h}^{2} / 2\langle\phi\rangle^{2}=0.129$ leads to a vanishing $\lambda_{2}$, which entails a diverging $m_{Z^{\prime}}$ as well as $\mu_{\mathrm{GW}}$. This leads to a departure from the perturbative domain of the Hempfling model and explains the excluded central regions in the plots in Fig. 4. The observed minimum $Z^{\prime}$ mass follows immediately from (4.20) for a vanishing $m_{\mathrm{PGB}}$ to be

$$
\begin{aligned}
m_{Z^{\prime}}^{\min } & =\sqrt[4]{\left(12 m_{t}^{4}-6 m_{W}^{2}-3 m_{Z}^{4}-m_{h}^{4}\right) / 3} \\
& =240.95 \mathrm{GeV}
\end{aligned}
$$

which is reproduced in the data. Below this value, we have a negative $m_{\mathrm{PGB}}$ and hence no second minimum.

In summary, we conclude that the Hempfling model in the GW scenario $\mathrm{B}$ gives rise to a perturbatively stable conformal extension of the SM all the way up to the Planck scale. The allowed values of $m_{Z^{\prime}}$ and the new scalar resonance $m_{\mathrm{PGB}}$ take a lens shape with the range

$$
\begin{aligned}
240 \mathrm{GeV} & <m_{Z^{\prime}}<1600 \mathrm{GeV}, \\
0 \mathrm{GeV} & <m_{X}<250 \mathrm{GeV} ;
\end{aligned}
$$

see Fig. 5. These values are to be contrasted to the quantum potential result in (3.12). 

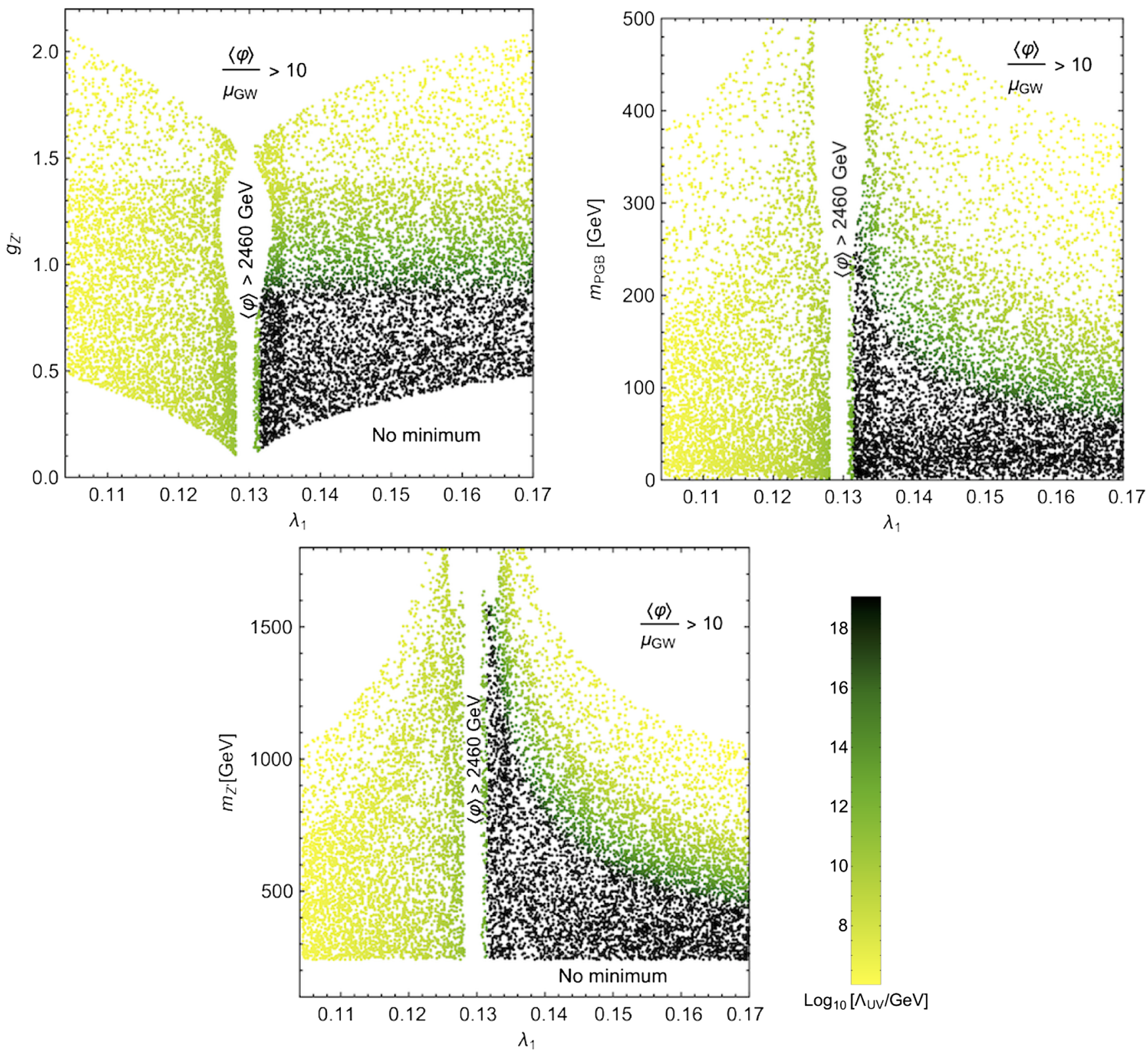

FIG. 4. Largest possible UV scales in the GW scenario B of the Hempfling model where the Higgs is not the pseudo-Goldstone boson (PGB) particle. A random set of 12000 dials of $\left(\lambda_{1}, g_{Z^{\prime}}\right)$ in the realm $\lambda_{1} \in[0.1,0.17]$ and $g_{Z^{\prime}} \in[0, \pi]$ were performed and tested for perturbative viability, and the UV breakdown scale of every point was computed. The nonsmooth jump at $\lambda_{1} \sim 0.13$ from a UV cutoff at the Planck scale to around $10^{12} \mathrm{GeV}$ is due to the onset of vacuum instability (negative $\lambda_{1}$ or $\lambda_{2}$ ) at that intermediate scale.

\section{SPECIAL CASE: SINGLE SCALAR SM EXTENSION $\left(g_{Z^{\prime}}=0\right)$}

If we set $g_{Z^{\prime}}=0$, i.e., we decouple the dark $Z^{\prime}$, we arrive at a single scalar extension of the SM Lagrangian. This model was studied in Ref. [8], and a set of couplings which led to a minimum of the one-loop effective potential resembling the standard model vacuum was reported. This calculation minimized the sum of the tree and oneloop effective potential without enforcing a hierarchy of couplings. This is problematic as the absence of the QP hierarchy assumption leads to the gauge dependence of physical data extracted from the effective potential; see Fig. 6. The explicit variation of the mass and the minimum value of the potential in the setup of Ref. [8] with the gauge parameter $\xi$ is manifest.

However, using the results of the previous sections, it can be seen that, independently of the choice of hierarchy, the single scalar conformal standard model without a gauge field does not allow for a stable vacuum consistent with experimental bounds. 


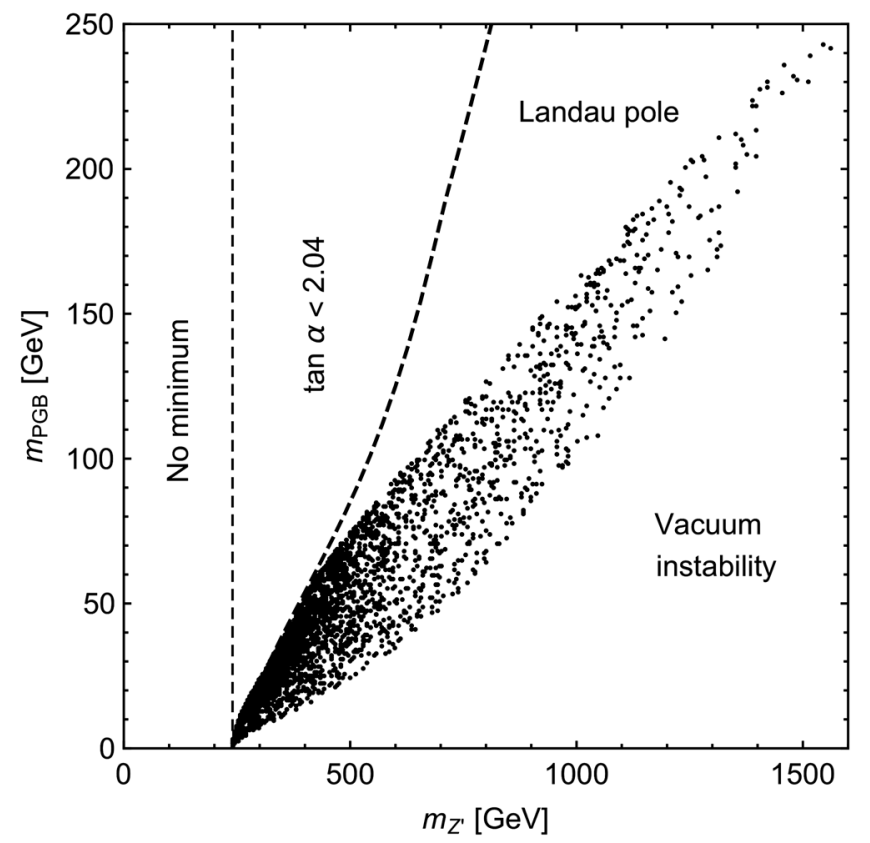

FIG. 5. Allowed mass ranges for the new scalar and $Z^{\prime}$ particles in the Gildener-Weinberg scenario B which are perturbatively stable up to the Planck scale.

First, look at the QP hierarchy $\lambda_{i} \sim g_{j}^{4}$. In the limit of vanishing $g_{Z^{\prime}}$, the mass matrix (3.5) for the scalar field becomes

$$
M_{i j}^{2}=\left(\begin{array}{cc}
-\frac{m_{0}^{4}}{8 \pi^{2}\langle\phi\rangle^{2}}-\lambda_{12}\langle S\rangle^{2} & \lambda_{12}\langle S\rangle\langle\phi\rangle \\
\lambda_{12}\langle S\rangle\langle\phi\rangle & -\lambda_{12}\langle\phi\rangle^{2}
\end{array}\right) .
$$

Both mass eigenvalues are real if and only if both the trace and the determinant of this matrix are non-negative. This leads to

$$
0 \leq \frac{\lambda_{12} m_{0}^{4}}{8 \pi^{2}}, \quad 0 \leq-\frac{m_{0}^{4}}{8 \pi^{2}\langle\phi\rangle^{2}}-\lambda_{12}\left(\langle S\rangle^{2}+\langle\phi\rangle^{2}\right)
$$

Recall that $m_{0}^{4}=(319 \mathrm{GeV})^{4}$ is a positive number. Therefore, the first condition can only be met if $\lambda_{12} \geq 0$. But then the second condition is violated, leading us to conclude that within this hierarchy there is no choice of couplings for which both mass eigenvalues turn out positive.

Now, we turn to the GW scenario with $\lambda_{i} \sim g_{j}^{2}$. For this assumption, we discussed two possible cases: The PGB of scale invariance could be identified with either the Higgs or the new scalar.

For the first case, there are no regions of the perturbative parameter space in which the mixing constraint

$$
\sin \alpha \leq 0.44
$$

for the mixing angle between $\phi$ and $S$ can be satisfied. This argument is independent of the value of $g_{Z^{\prime}}$ and stays valid in the decoupling limit.

In the second case, on the other hand, the formula for the mass of the new scalar (4.13) with vanishing $m_{Z^{\prime}}$ reads

$$
m_{X}^{2}=\frac{6 m_{W}^{4}+m_{Z}^{4}-12 m_{t}^{4}+m_{h}^{4}}{8 \pi^{2}\langle\varphi\rangle^{2}}<0
$$

and predicts a negative mass squared, implying that there is no stable minimum of the effective potential. Hence, this model is ruled out.

\section{CONCLUSIONS}

Since the seminal paper by Coleman and Weinberg [28], it has been an attractive theoretical concept that mass scales could be generated via quantum corrections to a classically scale-free model. Even more appealing is the scenario of such a mechanism underlying electroweak symmetry breaking in nature, which has been of considerable interest in the past; see e.g., Refs. [6-15]. The central task here is to establish a perturbatively consistent and gauge invariant formalism to minimize the effective potentials of such conformally extended standard models. In this paper, we have compared the two prevailing approaches in the

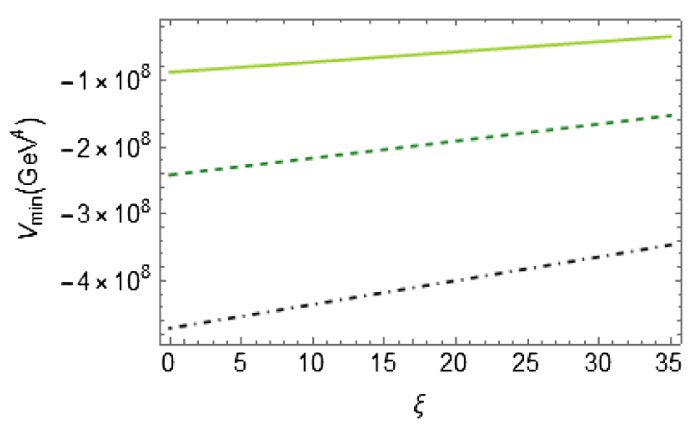

(a) Minimum of the effective potential

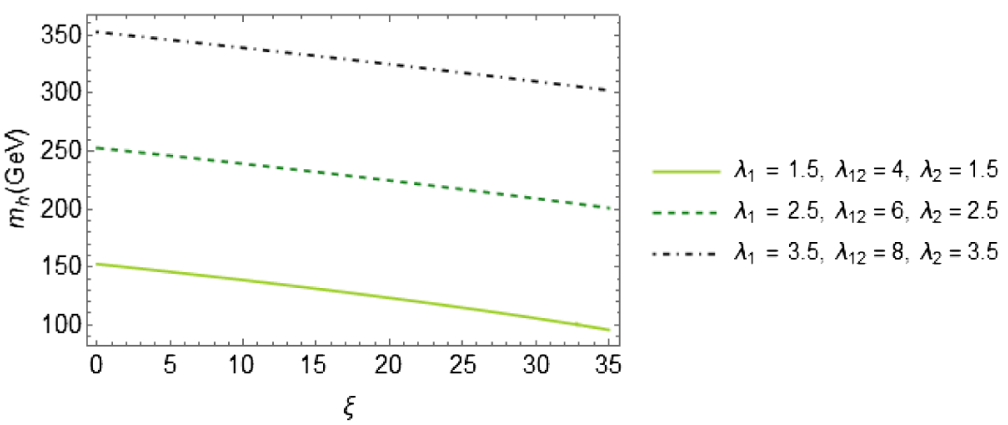

(b) Effective mass of the scalar field

FIG. 6. Dependence of the minimum value of the effective potential and effective Higgs mass on the gauge parameter $\xi_{B}=\xi_{W}=$ $\xi_{Z^{\prime}}=\xi$ in the standard model extended by a single scalar field without enforcing a certain hierarchy of couplings. 
literature in a simple model: the original ColemanWeinberg approach in its refined QP formulation and the GW method [21], which was designed to circumvent the multiple-scale problem of the Coleman-Weinberg approach in the multiscalar case. The central message of our work is that they are inequivalent as they inherently build on different scaling assumptions for the scalar and gauge/ Yukawa couplings. While this might be a posteriori unsurprising, it has not been appreciated adequately in the phenomenological literature, in which the focus is on the models and not on the method of minimizing the effective potential.

Each of the two scenarios has its own justification. The Gildener-Weinberg method represents the established framework for studying multiscalar effective potentials and requires the ordinary loop hierarchy of coupling constants $\lambda_{s} \sim g_{i}^{2}$. On the other hand, recent results on gauge invariance in the context of the standard model motivate the alternative hierarchy assumption $\lambda_{s} \sim g_{i}^{4}$, which we dubbed the quantum potential approach. These two different scalings may be thought of as alternative "sectors" or phases of a given model. Here, we emphasize that it is not sufficient to study only one sector (one scaling assumption) in order to exclude a model by phenomenological data. In addition, we have shown that both the QP and the GW methods yield gauge invariant results, at least to the one-loop order.

If different methods yield different phenomenological predictions, which approach should one use? Theoretically speaking, the answer is clear: The scaling pattern of the couplings determines the method to use. However, in the model building for conformally extended standard models, the scalar couplings $\lambda_{s}$ are part of the undetermined parameters. Hence, a thorough analysis of a given model necessitates the application of both methods and studying their phenomenological consequences. In practice, however, the situation is further complicated by the need to overcome the multiple-scale problem in multiscalar potentials within the QP approach.

In order to disentangle these two issues ( $\mathrm{QP}$ vs $\mathrm{GW}$ approaches and the multiscale problem), we studied a simple enough conformal extension of the SM, where the multiscale issue is delayed to the two-loop order. This is the Hempfling model, which represents the historically

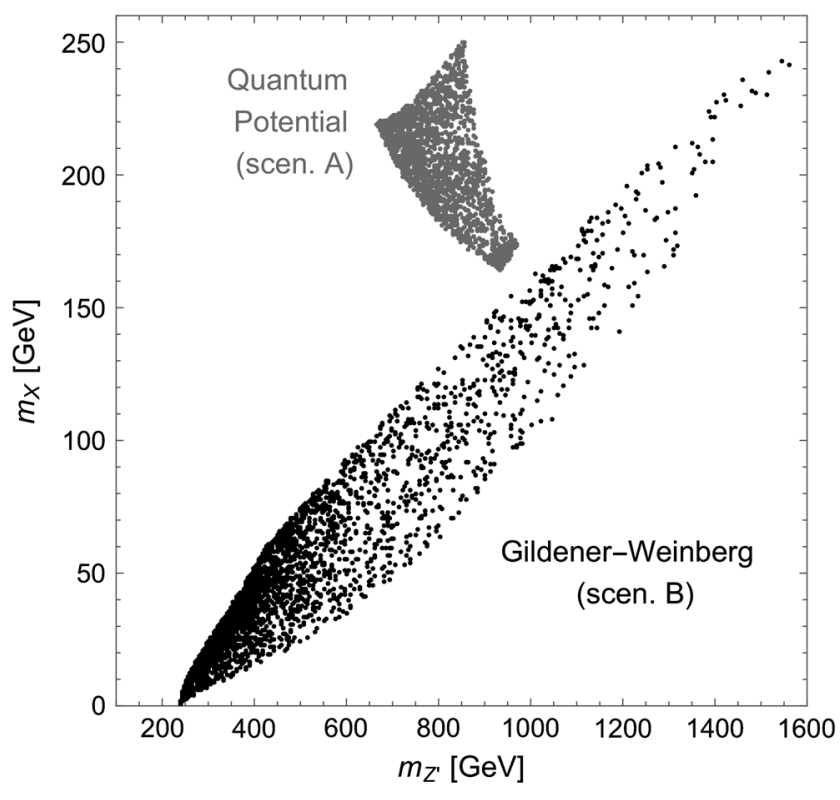

FIG. 7. Comparison of GW and QP approaches for the masses of the new scalar and $Z^{\prime}$ boson in the Hempfling model. Only data points with a UV cutoff at the Planck scale are plotted. Obviously, there is no overlap.

first phenomenological example of a conformal standard model extension. In addition to the standard model field content, this theory includes a new scalar and a new U(1) gauge field without kinematic mixing. As argued in Sec. V, a further reduction of the field content does not result in a phenomenologically viable model. Hence, in this sense, the Hempfling model is minimal. In particular, we explicitly determined and compared the allowed ranges for the new masses and coupling constants, which are compatible with experimental constraints on the scalar mixing angle and the absence of Landau poles and instabilities up to the Planck scale. Notably, both the QP and GW methods yield a stable and perturbatively consistent conformal modification of the standard model all the way up to the Planck scale, which reproduces the correct Higgs mass.

It turns out that the allowed combined mass regions for the new particles have no overlap; see Fig. 7. Still, the allowed intervals for the individual masses of the new gauge field and scalar are not disjoint:
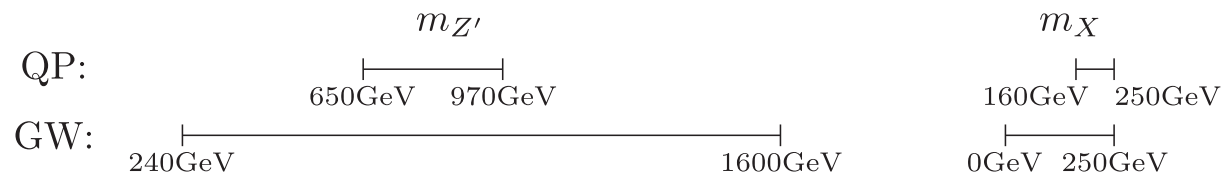

Hence, the obtained results at least indicate in which mass regions new particles should be expected in order to realize the generation of the electroweak scale by conformal symmetry breaking. 
We emphasize that, as opposed to the much simpler original Coleman-Weinberg model, our hierarchy assumptions have not been proven. Moreover, an analogous reasoning that relies on analytic solutions to the RG equations seems currently out of reach. It would thus be interesting to explore the consequences of alternative hierarchies, in which e.g., only one of the scalar couplings $\lambda_{i}$ is of the order $g_{j}^{4}$. It might also be interesting to draw connections to the functional renormalization group, cf. e.g., Ref. [29].

A very important point is the extension of our analysis to higher loop orders. While our prescription for the Hempfling model effectively results in a one-scale problem at one-loop order, extending the QP approach to higher loops will most likely face the problem of multiscale renormalization. This is a clear advantage of the GW method, which avoids this problem by construction. On the other hand, this fact does not necessarily imply the correctness of the latter approach for generic models. It would thus be important to continue and extend the presented comparison between different methods for the extraction of phenomenological data from effective potentials. Notably, various different ways of treating perturbative calculations with multiple scales have been proposed in the past; see e.g., Refs. [17,18,20-22]. Very recently, a new method was suggested in Ref. [22] and assumes the existence of a renormalization scale $\mu_{\mathrm{CPSS}}(\phi)$, where all loop corrections to the effective potential vanish. The vacuum is then obtained by minimizing the tree-level potential $V^{(0)}=\lambda_{i}\left(\mu_{\text {CPSS }}(\phi)\right) \phi^{4}$ with running couplings. It would be very interesting to compare all techniques in a two-loop computation in the context of a simple model. This should help to evaluate the methods' compatibility, perturbative consistency, and gauge independence.

While qualitatively pointing at a problem in the identification of phenomenological models via perturbative methods, quantitatively our analysis is certainly incomplete with regard to the gravitational interaction. Importantly, gravity introduces the Planck scale of which the naive treatment immediately violates conformal symmetry. Still, proposals for conformal extensions of the standard model exist and include the dynamic generation of the Planck scale; see e.g., Refs. [30]. Moreover, coupling the standard model to gravity necessarily induces higher interaction vertices of the scalar fields, even at the one-loop level [31]. These modify the effective potential and should thus be incorporated into an analysis to draw further conclusions on the viability of the considered models.

\section{ACKNOWLEDGMENTS}

We thank the CERN Theory Group for kind hospitality during the completion of this work. The work of F. L. is funded by the Deutsche Forschungsgemeinschaft (DFG, German Research Foundation), Project No. 363895012.

Note added.-Simultaneously with the current paper, Ref. [32], which has some overlaps with the ideas of our article and contains interesting complementary results, appeared on the arXiv.

\section{APPENDIX: PHENOMENOLOGICAL DATA}

Let us collect the phenomenological data which we use throughout the paper [33]. The masses of the gauge bosons are

$$
\begin{aligned}
m_{W} & =80.385 \pm 0.015 \mathrm{GeV}, \\
m_{Z} & =91.1876 \pm 0.0021 \mathrm{GeV}
\end{aligned}
$$

the top mass is

$$
m_{t}=173.1 \pm 0.6 \mathrm{GeV} \text {; }
$$

and the mass and vacuum expectation value of the Higgs take the values

$$
\begin{aligned}
& m_{H}=125.09 \pm 0.24 \mathrm{GeV}, \\
& \langle\phi\rangle=246.21971 \pm 0.00006 \mathrm{GeV} .
\end{aligned}
$$

The quartic Higgs coupling $\lambda$ is not known from experiment, but in the standard model, it can be deduced from the values for $m_{H}$ and $\langle\phi\rangle$. In extensions of the standard model, this relationship will necessarily be modified; in any case, $\lambda$ is not an independent input parameter in the models considered. Since in all our extensions it is still only one Higgs doublet which couples to the gauge bosons, we assume that $\langle\phi\rangle$ as given in (A3) stays the correct expectation value for the interaction eigenstate, even in cases in which the mass eigenstate of the Higgs boson differs by some mixing with another scalar. For the renormalization group evolution, we use the initial values [2]

$$
\begin{array}{rlrl}
g_{1}\left[m_{t}\right] & =0.3583, & & g_{2}\left[m_{t}\right]=0.64779, \\
g_{3}\left[m_{t}\right]=1.1666, & y_{t}\left[m_{t}\right]=0.9369
\end{array}
$$

at the $m_{t}$ scale. 
[1] G. Degrassi, S. Di Vita, J. Elias-Miro, J. R. Espinosa, G. F. Giudice, G. Isidori, and A. Strumia, Higgs mass and vacuum stability in the Standard Model at NNLO, J. High Energy Phys. 08 (2012) 098.

[2] D. Buttazzo, G. Degrassi, P. P. Giardino, G. F. Giudice, F. Sala, A. Salvio, and A. Strumia, Investigating the nearcriticality of the Higgs boson, J. High Energy Phys. 12 (2013) 089.

[3] S. R. Coleman and E. J. Weinberg, Radiative corrections as the origin of spontaneous symmetry breaking, Phys. Rev. D 7, 1888 (1973).

[4] M. Sher, Electroweak Higgs potentials and vacuum stability, Phys. Rep. 179, 273 (1989).

[5] W. A. Bardeen, On naturalness in the standard model, in Ontake Summer Institute on Particle Physics Ontake Mountain, Japan, 1995.

[6] J. P. Fatelo, J. M. Gerard, T. Hambye, and J. Weyers, Symmetry Breaking Induced by Top Loops, Phys. Rev. Lett. 74, 492 (1995); T. Hambye, Symmetry breaking induced by top quark loops from a model without scalar mass, Phys. Lett. B 371, 87 (1996).

[7] R. Hempfling, The Next-to-minimal Coleman-Weinberg model, Phys. Lett. B 379, 153 (1996).

[8] K. A. Meissner and H. Nicolai, Conformal symmetry and the Standard Model, Phys. Lett. B 648, 312 (2007).

[9] W.-F. Chang, J. N. Ng, and J. M. S. Wu, Shadow Higgs from a scale-invariant hidden U(1)(s) model, Phys. Rev. D 75, 115016 (2007); R. Foot, A. Kobakhidze, and R. R. Volkas, Electroweak Higgs as a pseudo-Goldstone boson of broken scale invariance, Phys. Lett. B 655, 156 (2007); R. Foot, A. Kobakhidze, K. L. McDonald, and R. R. Volkas, A Solution to the hierarchy problem from an almost decoupled hidden sector within a classically scale invariant theory, Phys. Rev. D 77, 035006 (2008); S. Iso, N. Okada, and Y. Orikasa, Classically conformal $B^{-} \mathrm{L}$ extended Standard Model, Phys. Lett. B 676, 81 (2009); The minimal B-L model naturally realized at TeV scale, Phys. Rev. D 80, 115007 (2009).

[10] L. Alexander-Nunneley and A. Pilaftsis, The minimal scale invariant extension of the Standard Model, J. High Energy Phys. 09 (2010) 021.

[11] C. D. Carone and R. Ramos, Classical scale-invariance, the electroweak scale and vector dark matter, Phys. Rev. D 88, 055020 (2013); C. Englert, J. Jaeckel, V. V. Khoze, and M. Spannowsky, Emergence of the electroweak scale through the Higgs portal, J. High Energy Phys. 04 (2013) 060.

[12] A. Farzinnia, H.-J. He, and J. Ren, Natural electroweak symmetry breaking from scale invariant Higgs mechanism, Phys. Lett. B 727, 141 (2013).

[13] T. Hambye and A. Strumia, Dynamical generation of the weak and dark matter scale, Phys. Rev. D 88, 055022 (2013); M. Heikinheimo, A. Racioppi, M. Raidal, C. Spethmann, and K. Tuominen, Physical naturalness and dynamical breaking of classical scale invariance, Mod. Phys. Lett. A 29, 1450077 (2014); M. Holthausen, J. Kubo, K. S. Lim, and M. Lindner, Electroweak and conformal symmetry breaking by a strongly coupled hidden sector, J. High Energy Phys. 12 (2013) 076; C. T. Hill, Is the Higgs boson associated with Coleman-Weinberg dynamical symmetry breaking?, Phys. Rev. D 89, 073003 (2014);
A. Karam and K. Tamvakis, Dark matter and neutrino masses from a scale-invariant multi-Higgs portal, Phys. Rev. D 92, 075010 (2015); S. Oda, N. Okada, and D.-s. Takahashi, Classically conformal $U(1)^{\prime}$ extended standard model and Higgs vacuum stability, Phys. Rev. D 92, 015026 (2015); A. Das, S. Oda, N. Okada, and D.-s. Takahashi, Classically conformal U(1)? extended standard model, electroweak vacuum stability, and LHC Run-2 bounds, Phys. Rev. D 93, 115038 (2016).

[14] A. J. Helmboldt, P. Humbert, M. Lindner, and J. Smirnov, Minimal conformal extensions of the Higgs sector, J. High Energy Phys. 07 (2017) 113.

[15] T. Hambye, A. Strumia, and D. Teresi, Super-cool dark matter, J. High Energy Phys. 08 (2018) 188.

[16] O. Antipin, M. Mojaza, and F. Sannino, Conformal extensions of the Standard Model with Veltman conditions, Phys. Rev. D 89, 085015 (2014); P. H. Chankowski, A. Lewandowski, K. A. Meissner, and H. Nicolai, Softly broken conformal symmetry and the stability of the electroweak scale, Mod. Phys. Lett. A 30, 1550006 (2015).

[17] M. B. Einhorn and D. R. T. Jones, A new renormalization group approach to multiscale problems, Nucl. Phys. B230, 261 (1984).

[18] C. Ford, Multiscale renormalization group improvement of the effective potential, Phys. Rev. D 50, 7531 (1994); C. Ford and C. Wiesendanger, A Multiscale subtraction scheme and partial renormalization group equations in the $\mathrm{O}(\mathrm{N})$ symmetric $\varphi^{4}$ theory, Phys. Rev. D 55, 2202 (1997); Multiscale renormalization, Phys. Lett. B 398, 342 (1997).

[19] J. A. Casas, V. Di Clemente, and M. Quiros, The Effective potential in the presence of several mass scales, Nucl. Phys. B553, 511 (1999).

[20] M. Bando, T. Kugo, N. Maekawa, and H. Nakano, Improving the effective potential: Multimass scale case, Prog. Theor. Phys. 90, 405 (1993).

[21] E. Gildener and S. Weinberg, Symmetry breaking and scalar bosons, Phys. Rev. D 13, 3333 (1976).

[22] L. Chataignier, T. Prokopec, M. G. Schmidt, and B. Swiezewska, Single-scale renormalisation group improvement of multi-scale effective potentials, J. High Energy Phys. 03 (2018) 014.

[23] R. Jackiw, Functional evaluation of the effective potential, Phys. Rev. D 9, 1686 (1974); L. Dolan and R. Jackiw, Gauge invariant signal for Gauge symmetry breaking, Phys. Rev. D 9, 2904 (1974);

[24] A. Andreassen, W. Frost, and M. D. Schwartz, Consistent use of effective potentials, Phys. Rev. D 91, 016009 (2015).

[25] A. Andreassen, W. Frost, and M. D. Schwartz, Consistent Use of the Standard Model Effective Potential, Phys. Rev. Lett. 113, 241801 (2014).

[26] M. Srednicki, Quantum Field Theory (Cambridge University Press, Cambridge, England, 2007).

[27] A. Farzinnia and J. Ren, Higgs partner searches and dark matter phenomenology in a classically scale invariant Higgs boson sector, Phys. Rev. D 90, 015019 (2014).

[28] S. R. Coleman and E. J. Weinberg, Radiative corrections as the origin of spontaneous symmetry breaking, Phys. Rev. D 7, 1888 (1973). 
[29] H. Gies and R. Sondenheimer, Renormalization group flow of the Higgs potential, Phil. Trans. R. Soc. A 376, 20170120 (2018).

[30] I. Bars, P. Steinhardt, and N. Turok, Local conformal symmetry in physics and cosmology, Phys. Rev. D 89, 043515 (2014); P. G. Ferreira, C. T. Hill, and G. G. Ross, Inertial spontaneous symmetry breaking and quantum scale invariance, Phys. Rev. D 98, 116012 (2018); M. Shaposhnikov and A. Shkerin, Conformal symmetry: towards the link between the Fermi and the Planck scales, Phys. Lett. B 783, 253 (2018).
[31] F. Loebbert and J. Plefka, Quantum gravitational contributions to the Standard Model effective potential and vacuum stability, Mod. Phys. Lett. A 30, 1550189 (2015).

[32] L. Chataignier, T. Prokopec, M. G. Schmidt, and B. Swiezewska, Systematic analysis of radiative symmetry breaking in models with extended scalar sector, J. High Energy Phys. 08 (2018) 083.

[33] C. Patrignani et al. (Particle Data Group Collaboration), Review of particle physics, Chin. Phys. C 40, 100001 (2016). 